\title{
Acute Chest Infections in Children
}

\author{
C. Owens \\ Department of Radiology, Great Ormond Street Hospital for Children, NHS Trust, London, UK
}

\section{Introduction}

Radiation dose is a contentious issue in pediatrics, as it is well-established that the lifetime cancer mortality risks attributable to CT examinations are considerably higher than for adults. As proposed by the ALARA principle 'as low as reasonably achievable', the selection of appropriate scanning parameters focuses on the optimization of image quality whilst delivering the lowest possible radiation dose and shifting the risk-benefit balance towards benefit.

The technical parameters that need to be selected for any scan include collimation thickness and tube current (milliamperage, and kilovoltage). The collimation thickness is the minimum section thickness that can be acquired once the scan is finished, and in a 16-row MDCT scanner is usually $1.5 \mathrm{~mm}$. Thinner collimation $(0.75$ $\mathrm{mm}$ ) increases the radiation dose by approximately $30 \%$ with our in-house reduced protocol and is applied only in selected cases of vascular abnormalities, visualization of small structures, in cardiac CTs, and when high-resolution thin-slice parenchymal lung imaging is required for detailed assessment of the lung parenchyma (Tables 1-3).

Table 2. High-resolution CT chest scanning parameters according to child's weight

\begin{tabular}{lccc}
\hline & $<15 \mathrm{~kg}$ & $<30 \mathrm{~kg}$ & $>30 \mathrm{~kg}$ \\
\hline $\mathrm{kVp}$ & 100 & 100 & 100 \\
eff. mAs & 20 & 30 & 55 \\
Collimation (mm) & 1 & 1 & 1 \\
Scan slice width (mm) & 1 & 1 & 1 \\
Table-feed (mm) & 10 & 10 & 10 \\
Scan time (s) & 0.36 & 0.75 & 0.75 \\
CTDI (mGy) & 0.21 & 0.32 & 0.59 \\
\hline
\end{tabular}

Table 1. Volumetric CT chest scanning parameters (16 slice Somaton Scanner) according to child's weight when a routine (1.5-mm collimation) or 'Combiscan' $(0.75-\mathrm{mm})$ protocol is used

\begin{tabular}{|c|c|c|c|c|c|c|c|c|c|c|}
\hline & \multicolumn{2}{|c|}{$<15 \mathrm{~kg}$} & \multicolumn{2}{|c|}{$15-24 \mathrm{~kg}$} & \multicolumn{2}{|c|}{$25-34 \mathrm{~kg}$} & \multicolumn{2}{|c|}{$35-44 \mathrm{~kg}$} & \multicolumn{2}{|c|}{$45-55 \mathrm{~kg}$} \\
\hline & Volume & Combi & Volume & Combi & Volume & Combi & Volume & Combi & Volume & Combi \\
\hline $\mathrm{kVp}$ & 100 & 100 & 100 & 100 & 100 & 100 & 100 & 100 & 100 & 100 \\
\hline eff. mAs & 20 & 20 & 25 & 25 & 35 & 35 & 55 & 55 & 75 & 75 \\
\hline Collimation (mm) & 1.5 & 0.75 & 1.5 & 0.75 & 1.5 & 0.75 & 1.5 & 0.75 & 1.5 & 0.75 \\
\hline Scan slice width (mm) & 5 & 5 & 5 & 5 & 5 & 5 & 5 & 5 & 8 & 8 \\
\hline Table-feed (mm) & 24 & 12 & 24 & 12 & 24 & 12 & 24 & 12 & 24 & 12 \\
\hline Scan time (s) & 0.5 & 0.5 & 0.5 & 0.5 & 0.5 & 0.5 & 0.5 & 0.5 & 0.5 & 0.5 \\
\hline CTDI (mGy) & 0.9 & 1.0 & 1.13 & 1.31 & 1.58 & 1.75 & 2.48 & 2.75 & 3.38 & 3.75 \\
\hline
\end{tabular}

Table 3. Dose comparison for different scanning protocols in a phantom study in our institution

\begin{tabular}{|c|c|c|c|c|c|c|c|c|c|c|}
\hline & \multicolumn{10}{|c|}{ Effective dose (mSv) } \\
\hline & \multicolumn{2}{|c|}{ Volume (1.5-mm) } & \multicolumn{2}{|c|}{ Combi $(0.75-\mathrm{mm})$} & \multicolumn{2}{|c|}{ HRCT } & \multicolumn{2}{|c|}{ CTA } & \multicolumn{2}{|c|}{ CXR } \\
\hline & $\mathrm{M}$ & $\mathrm{F}$ & $\mathrm{M}$ & $\mathrm{F}$ & $\mathrm{M}$ & $\mathrm{F}$ & M & $\mathrm{F}$ & AP & LAT \\
\hline$<15 \mathrm{~kg}$ & 0.77 & 0.90 & 0.9 & 1.05 & 0.36 & 0.42 & 1.30 & 1.51 & 0.00487 & 0.00799 \\
\hline $15-24 \mathrm{~kg}$ & 0.93 & 1.09 & 1.13 & 1.31 & 0.36 & 0.42 & 1.62 & 1.89 & 0.00874 & 0.01086 \\
\hline $25-34 \mathrm{~kg}$ & 1.34 & 1.56 & 1.58 & 1.84 & 0.54 & 0.63 & 2.24 & 2.62 & 0.01163 & 0.00968 \\
\hline $35-44 \mathrm{~kg}$ & 2.11 & 2.46 & 2.48 & 2.89 & 1.00 & 1.17 & 2.57 & 3.0 & 0.01769 & 0.01452 \\
\hline
\end{tabular}

$H R C T$, High-resolution computed tomography; $C T A$, CT angiography; $C X R$, chest X-ray 
Methods adopted to minimize the radiation dose during MDCT include:

1. Applying a dose modulation function in which the system samples the patient thickness and adjusts (e.g., reduces) the exposure accordingly when the tube is in the AP/PA position, as patients are narrower in AP than in the side-to-side orientation.

2. Reduction of the kilovoltage to $100 \mathrm{kVp}$ when imaging the thorax. Further reduction to $80 \mathrm{kVp}$ is possible for CT angiography (CTA), but as resolution of the parenchyma is not ideal this is applied only if lung pathology is unlikely.

3. If possible, selecting a tube collimation of $1.5 \mathrm{~mm}$. Although $0.7-5 \mathrm{~mm}$ collimation improves spatial resolution, it increases the radiation dose and is therefore reserved for CTA or in those cases in which thin slice reconstruction is indicated.

4. Selection of an appropriate mAs, dependent on the patient's weight or cross-sectional diameter.

Unlike the single-slice scanner, an increase or decrease in table-feed time for MDCT scanners only affects the overall scanning time. An increase in table speed results in a concomitant increase in $\mathrm{mA}$, which has no effect on the dose delivered. The tube current is automatically compensated to ensure that the preset effective and total $\mathrm{mAs}$ are delivered, i.e., a fast table movement results in an automatic increase in the $\mathrm{mA}$ while keeping the mAs constant.

\section{The Immunocompetent Child}

The roles played by imaging in the evaluation of community acquired pneumonias are multiple: confirmation or exclusion of pneumonia, characterization and attempts to predict the infectious agent, exclusion of other causes of symptoms, evaluation when resolution is slow or incomplete, and evaluation of related complications, such as suspected lung abscess (or cavitatory necrosis). The latter is more common and more benign in children than was previously believed, with only $41 \%$ of CT-proven cases visible on chest X-ray (CXR). Moreover the prognosis with conservative management is much better than in adults and there is relatively little lung scarring. CT is also valuable in detecting the presence of superinfected bronchopulmonary foregut malformations or underlying/ensuing bronchiectasis.

\section{Mycobacterium tuberculosis}

In children, pulmonary TB usually occurs as a result of primary infection acquired from an infected adult. Disseminated or extrapulmonary tuberculosis is relatively rare in children, raises the possibility of underlying immunodeficiency, and is classified as an AIDS indicator.
The radiological features of pulmonary TB are usually similar to those in immunocompetent individuals and include lobar consolidation, segmental or lobar atelectasis, pleural effusions, and lymphadenopathy. Pulmonary cavitation is uncommon. Large-volume lymphadenopathy is a frequent finding, the paratracheal and hilar regions being the most common sites. Occasionally, the chest radiograph appears normal. Miliary disease is uncommon, although when it occurs it may be indistinguishable from lymphocytic interstitial pneumonia.

Contrast-enhanced CT (CECT) and high-resolution CT (HRCT) are of particular value in the confirmation of mediastinal and miliary disease. Mediastinal lymphadenopathy is often large in volume; it typically demonstrates prominent peripheral enhancement following intravenous contrast. The classical HRCT feature of miliary tuberculosis is a reticulonodular infiltrate. In adults, the nodules are typically uniform in size, measuring 1-3 mm. However, in children, nodules are more varied in size and definition than described by the classic Fleischner criteria, which are based on the adult form of the disease.

\section{The Immunocompromised Child}

Immunodeficiency states in children may be sub-divided into two major groups; congenital (primary) and acquired (secondary). The spectrum of illness and imaging appearances are similar regardless of the underlying cause of immunodeficiency. All immunodeficiency states are associated with an increased susceptibility to infection and neoplasia, with lymphoproliferative disorders being the most frequent. However, the type of infection encountered and the risk of neoplasia are influenced by the underlying defect (whether predominantly humoral or cell-mediated), the use of immunosuppressive drugs or radiotherapy and the length of immunosuppression. A working knowledge of the underlying likely defect is therefore important when interpreting imaging functions in children with immunodeficiency states.

Primary, or congenital, immunodeficiency disorders are an inherited group of diseases resulting from innate defects of the immune system. Secondary or acquired immunodeficiency disorders occur as a consequence of infection with the human immunodeficiency virus (HIV), or secondary to immunosuppressive drug therapy or chemoradiotherapy (for hematological/solid-organ malignancies or as preparation for bone-marrow transplantation). Both primary and secondary immunodeficiency states result in an increased susceptibility to infection, with the respiratory tract being the most common disease site. Certain complications, however, particularly infectious ones, are common to all immunodeficiency states and extensive overlap in imaging findings is observed. 


\section{Primary Immunodeficiency Disorders}

\section{Background}

Primary (congenital) immunodeficiency disorders (PID) represent a rare heterogeneous group of genetically determined disorders characterized by defects of cell-mediated immunity, antibody production, and/or the complement and phagocyte systems (Table 4).

Clinical manifestations vary according to the subtype of PID and include recurrent infections, infection with unusual or opportunistic organisms, failure to thrive, skin rashes, recurrent skin sepsis, and unusual wound healing. A family history of recurrent infections or premature death among siblings may also be revealed. Perhaps the most important, and frequent, clinical manifestation of PIDs is recurrent sinopulmonary infections which, if not adequately treated, may result in the development of obstructive lung disease, chronic respiratory failure, and ultimately premature death.

After infection, malignancy is the second leading cause of death. The lymphoproliferative disorders (LPDs) account for the majority of tumors, with Epstein-Barr virus being associated in approximately $30-60 \%$ of cases. The risk of developing a malignancy ranges from 1 to $25 \%$. The children at greatest risk are those with Wiscott-Aldrich syndrome and combined variable immunodeficiency.

\section{Pulmonary Complications}

\section{Diffuse Lung Disease}

Common to all the primary immunodeficiency disorders is an inability to mount a normal or adequate antibody re-

Table 4. Immunodeficiency disorders

\begin{tabular}{ll}
\hline Disorder & Inheritance \\
\hline Combined immunodeficiencies & \\
Severe combined immunodeficiency & $\mathrm{X}, \mathrm{AR}$ \\
Purine nucleosidase phosphorylase & $\mathrm{AR}$ \\
Common variable immunodeficiency & \\
Predominantly antibody deficiencies & \\
Selective Ig A deficiency & $\mathrm{AR}, \mathrm{AD}, \mathrm{S}$ \\
Selective Ig G subclass deficiency & $\mathrm{AR}, \mathrm{S}$ \\
Hyper Ig M & $\mathrm{X}, \mathrm{AR}$ \\
X-linked agammaglobulinemia & $\mathrm{X}$ \\
Well-defined immunodeficiencies & \\
Wiscott-Aldrich syndrome & $\mathrm{X}$ \\
Ataxia telangectasia & $\mathrm{AR}$ \\
DiGeorge syndrome & $\mathrm{AD}, \mathrm{S}$ \\
Other immunodeficiencies & \\
X-linked lymphoproliferative disease & $\mathrm{X}$ \\
Hyper IgE syndrome & $\mathrm{S}$ \\
Phagocytic defects & \\
Chediak-Higashi syndrome & $\mathrm{AR}$ \\
\hline
\end{tabular}

$A R$, Autosomal recessive; $A D$, autosomal dominant; $S$, sporadic; $X$, X-linked sponse to antigenic stimulation, resulting in susceptibility to bacterial infections, particularly of the respiratory tract. Bronchiectasis is a common complication, occurring in $20-40 \%$ of patients.

Typical radiographic features include hyperinflation and bronchial-wall thickening, with or without dilation. The chest radiograph is, nevertheless, insensitive and commonly appears normal or shows only subtle change. HRCT detects abnormalities not visible on the plain, thus improving diagnostic accuracy compared to the CXR. Moreover, it allows for an earlier diagnosis.

\section{Pneumocystis carinii Pneumonia}

Pneumonia caused by Pneumocystis carinii usually occurs in children whose primary immunodeficiency is undiagnosed and, indeed, is frequently the first indicator of an underlying immunodeficiency state. Classical radiographic appearances include hyperinflation with diffuse bilateral interstitial or nodular infiltrates that may be subtle initially but rapidly progress to widespread alveolar shadowing.

\section{Angio-invasive Aspergillosis}

The typical manifestation of angio-invasive pulmonary aspergillosis (IPA) consists of multi-focal areas of parenchymal inflammation caused by hematogenous dissemination of the organisms. IPA is associated with infarction and necrosis secondary to vascular obstruction.

Classical radiographic features include either solitary or multiple nodules or masses, with or without cavitation. In some cases, however, the chest radiograph appears normal or may demonstrate a focal infiltrate indistinguishable from a pyogenic pneumonia. Specific HRCT features have been described, probably the most characteristic being a 'halo' of ground-glass attenuation representing peri-lesional necrosis and hemorrhage, surrounding a central focal fungal nodule or infarct. A second commonly described feature of IPA is lesional cavitation with the formation of an air crescent. Occasionally, IPA manifests as a necrotizing pneumonia, with infiltration of local structures or organs. Although these classical features have been described, in many cases the imaging appearances are non-specific.

\section{Secondary (Acquired) Immunodeficiencies}

\section{Immunodeficiency Secondary to Immunosuppressive or Myeloablative Chemoradiotherapy}

Respiratory complications may be sub-divided into two broad categories: infectious and noninfectious. The frequency, range, and severity of pulmonary complications are influenced by the intensity and length of immunosuppression and are also dependent on whether the treatment goal is immunosuppression or myeloablation. 


\section{Stem-Cell Transplantation}

Stem-cell transplantation (SCT) is followed by a temporal and predictable sequence of events. Initially, there is a marked transient neutropenia with neutrophil numbers returning to normal within 2-4 weeks. By contrast, lymphocyte recovery is more prolonged, with absolute numbers taking approximately 3 months to return to normal. Despite the recovery of lymphocyte numbers, cellular (B and T cell) and humoral (particularly antiobody production) immunity usually remains impaired for a further 6-12 months, and antibody production can take up to a year to return to normal.

The development of graft-versus-host disease (GVHD) and the resultant requirement for increased immunosuppression also delays immunological recovery and is associated with an increased risk of infectious pulmonary complications.

Pulmonary complications occur in $40-60 \%$ of patients following SCT and are responsible for $10-40 \%$ of transplant-related deaths. The type of pulmonary complication will be influenced by the type of underlying immune defect and may be subdivided into early (up to 100 days) and late (after 100 days) complications.

\section{Early Pulmonary Complications}

\section{Infectious Complications}

Within the early post-transplant period, the most frequently infecting organisms are bacteria and fungi, especially gram-negative organisms and Aspergillus sp. Children are also at risk for certain viral infections, the most important being respiratory syncytial virus (RSV) and herpes simplex virus (HSV).

Bacterial pneumonias occur in approximately $10 \%$ of patients during the pre-engraftment period and are associated with a high mortality.

Viral pneumonias, due, for example, to RSV, HSV, adenovirus, and varicella-zoster virus (VZV), typically occur within the early post-transplant period. RSV infection commonly leads to a devastating viral pneumonia, with a mortality rate approaching 50\%.

Fungal pneumonia, (IPA) is reported to occur in approximately $4 \%$ of children undergoing SCT. Children are at particular risk during episodes of prolonged neutropenia, as are children receiving high-dose corticosteroids for the treatment of GVHD. The most common infecting organisms are Aspergillus fumigatus and Aspergillus flavus. Previously, the prognosis was dismal but the introduction of expensive albeit efficacious triple antifungal therapy has altered mortality rates in children. As a consequence of a profound neutropenia, the radiographic appearances of IPA may be atypical and, as a result, diagnosis is often delayed. A definitive diagnosis can be established only with certainty after histological confirmation of hyphae within lung tissue. Using EORTC criteria, the probability rates can be high and empirical therapy should be initiated in the presence of CT findings of multifocal nodules, perifissural consolidation, halo sign, and cavitation (which is probably less often observed in children than adults).

\section{Acute Noninfectious Pulmonary Complications}

Those occurring within the early post-transplant period include idiopathic pneumonia syndrome, pulmonary edema, pulmonary hemorrhage, pulmonary veno-occlusive disease, and relapse of the underlying malignancy.

Diffuse alveolar hemorrhage occurs in approximately $10 \%$ of patients undergoing allogeneic bone-marrow transplantation, usually at the time of engraftment. It is frequently associated with other pulmonary complications, particularly infection, and has a high mortality. The classical radiographic features are those of air space shadowing, which may be patchy, and multi-focal or more confluent consolidation with air bronchograms. Cessation of bleeding typically results in rapid clearing over a few days.

Idiopathic pneumonia syndrome is defined as diffuse lung injury for which no cause has been identified. GVHD and pre-transplant total body irradiation are contributing factors. It usually occurs 6-8 weeks following bone-marrow transplantation. Radiographic features are nonspecific and variable; they include diffuse air-space shadowing and/or interstitial infiltrates, often with a nodular component. They may be indistinguishable from adult respiratory distress syndrome (ARDS).

\section{Late Pulmonary Complications}

\section{Infectious Pulmonary Complications}

Patients continue to be at risk from bacterial infections even after engraftment, due to continuing defective humoral immunity. The most common infecting organisms are encapsulated bacteria such as Streptococcus pneumoniae and Haemophilus influenzae. Others include Pneumocystis carinii (particularly in those where antibiotic compliance is poor), cytomegalovirus and adenovirus.

\section{Non-infectious Pulmonary Complications}

These include obliterative bronchiolitis, diffuse alveolar damage, lymphocytic interstitial pneumonia, and relapse of the underlying disease.

Obliterative (constrictive) bronchiolitis (OB) is otherwise known as constrictive bronchiolitis and occurs secondarily to chronic pulmonary GVHD. OB is characterized by airflow limitation subsequent to submucosal and peribronchiolar inflammation, fibrosis and vascular sclerosis. It predominantly affects the medium-sized airways (respiratory bronchioles). Radiological features include hy- 
perinflation, bronchial-wall thickening and/or dilation. In the majority, however, the chest radiograph appears normal or shows only subtle changes. HRCT improves diagnostic accuracy compared to the chest radiograph. Classical HRCT findings are patchy areas of lung hypoattenuation associated with a reduction in the number and caliber of vessels, with or without associated bronchiectasis or bronchial-wall thickening.

\section{Solid-Organ Transplantation}

As a consequence of long-term immunosuppressive therapy, recipients of solid-organ transplants are at risk of recurrent infections as well as an increased incidence of neoplasia, lymphoproliferative disorders being the most frequent. The type of organ transplantation in addition to the type and intensity of immunosuppression regimen used will influence the pulmonary complications encountered.

\section{Post-transplant Lymphoproliferative Disorders}

These represent a spectrum of disease ranging from polyclonal lymphoid hyperplasia to monoclonal malignant lymphoma. The majority of post-transplant LPDs are of B-cell origin and are almost invariably associated with Epstein-Barr virus (EBV) infection. The incidence varies from $1 \%$ in renal transplant recipients to $10 \%$ in heart and heart/lung transplant recipients. The time between transplant and onset varies from 1 month to several years.

The radiographic appearances of intrathoracic LPD are variable and range from discrete parenchymal nodules, which may be solitary or multiple, to diffuse or focal reticulonodular infiltrates or consolidation. These findings may or may not be associated with mediastinal lymphadenopathy. Since imaging appearances may mimic pyogenic or fungal infection, biopsy is usually required.

\section{HIV-Associated Acquired Immunodefiency Syndrome}

The human immunodeficiency virus (HIV) produces its dominant effects on $\mathrm{T}$ cells, with CD4 cells being the main site of viral replication. The virus also infects CD4negative cells (B and T cells), resulting in defects in both cell-mediated and humoral immunity. Since the epidemic began, in the late 1980s over 3.8 million children have died of AIDS.

\section{Pulmonary Complications}

Pulmonary disease is the most common initial manifestation of HIV in children and is the primary cause of death in $50 \%$ of children with AIDS. The most common respiratory manifestations of HIV are bacterial pneumonias, Pneumocystis carinii pneumonia, and lymphocytic interstitial pneumonia.
Pneumocystis carinii pneumonia is frequently the initial manifestation of HIV, often presenting in early infancy. It is the most common opportunistic pulmonary infection in children with AIDS, occurring in up to $50 \%$, and is the leading pulmonary cause of death. Radiographic appearances are variable and include hyperinflation with diffuse bilateral interstitial of nodular infiltrates and widespread alveolar shadowing. Asymmetric, focal, or patchy infiltrates are also frequent. In contrast to the disease in children with primary immunodeficiency disorders, cavitary nodules and cysts are common and occur in up to onethird of patients. Lymphadenopathy and pleural effusions are uncommon and, if present, an alternative diagnosis, such as lymphocytic interstitial pneumonia or TB, should be considered. Pneumothoraces and/or pneumomediastinum are a frequent complication. HRCT findings include patchy or diffuse ground-glass opacity, consolidation, cysts or cavities, centrilobular opacities, nodules, and interlobular septal thickening.

Lymphocytic interstitial pneumonitis occurs in approximately one-third of infected children. It makes up part of the diffuse infiltrate lymphocytosis syndrome and is thought to represent a direct 'hyperimmune' lung response to the presence of either HIV or Epstein-Barr virus. The disease appears to be restricted to those children expressing HLA DR 5 alleles and is associated with a slower rate of AIDS progression. Radiographic appearances include interstitial reticulonodular infiltrates that may progress to patchy air-space consolidation. In contrast to Pneumocystis carinii pneumonia, lymphadenopathy is common and often becomes more prominent during episodes of super-added infection.

Viral infections may occur as a result of a primary infection or due to reactivation of latent virus. The infecting viruses include RSV, influenza and parainfluenza viruses, $\mathrm{CMV}$, and occasionally measles. Children infected with VZV often present with a more protracted illness than seen in immunocompetent children, and approximately $50 \%$ suffer from chronic or recurrent VZV infection. The risk of complications increases with increasing immunological impairment. The mortality rate in children with AIDS is $15 \%$. The chest radiograph in VZV pneumonia patients typically shows evidence of bilateral diffuse reticulonodular infiltrates. The nodules are range in size from 3 to $10 \mathrm{~mm}$; they often coalesce, resulting in focal areas of consolidation. Superimposed bacterial infection is common.

Mycobacterium tuberculosis may occur at any stage of HIV infection. As noted above, in children, it usually is the result of primary infection acquired from an infected adult. Disseminated or extrapulmonary tuberculosis is classified as an AIDS indicator in children. The radiological features are usually similar to those in immunocompetent controls and include lobar consolidation, segmental or lobar atelectasis, pleural effusions, and lymphadenopathy. Pulmonary 
cavitation is uncommon. Large-volume lymphadenopathy is a frequent finding, the paratracheal and hilar regions being the most common sites.

\section{Conclusions}

Pulmonary complications are common in immunocompetent and immunodeficient children and CXR remains the first method of examination. Sadly, tuberculosis has returned to challenge us and the radiologist must keep this in mind when analyzing radiographs that show abundant lymphadenopathy, or consolidation that does not resolve with conventional anti-microbial therapy. A respiratory presentation is the most common initial manifestation of immunodeficiency states in children, with infectious complications being the most common. There is overlap in the radiological findings, and correlation with the cause and severity of the underlying immunodeficiency state is important when interpreting imaging findings.
All immunodeficiency states are also associated with an increased incidence of neoplasia, particularly lymphoproliferative disorders, the chest being a frequent site of disease. The radiological manifestations in addition to the clinical presentations are often atypical and may be misinterpreted as infection. It is therefore vital that both clinicians and radiologists be aware of the protean manifestations of these potentially treatable conditions.

\section{Suggested Reading}

Lucaya J, Strife JL (eds) (2002) Paediatric Chest Imaging. Chest Imaging in Infants and Children. Springer-Verlag, Heidelberg

Owens CM, Veys PA (2002) Respiratory Infections following Haemopoetic Stem Cell Transplantation. In: Childhood Respiratory Infections: British Medical Bulletin Vol 61. Oxford University Press, Oxford

Webb WR, Muller NL, Naidich DP (2001) Disease characterised primarily by parenchymal opacification. In: High Resolution CT of the Lung, $3^{\text {rd }}$ edn. Lippincott-Williams \& Wilkins, Philadelphia, pp 355-420 